\title{
Effect of Electrode Resistance and Stray Capacitance on Measurement of Soil Properties
}

\author{
V. M. Gohil ${ }^{1}$ *, J. P. Pabari ${ }^{2}$, S. R. Panchal ${ }^{3}$ and V. H. Nayak ${ }^{4}$ \\ ${ }^{1}$ Sardar Vallabhbhai Patel Institute of Technology, Vasad, India. \\ ${ }^{2}$ Physical Research Laboratory, Ahmadabad, India \\ ${ }^{3}$ Sardar Vallabhbhai Patel Institute of Technology, Vasad, India. \\ ${ }^{4}$ Parul Institute of Technology, Waghodiya, Vadodara, India.
}

\begin{abstract}
The study of soil electromagnetic properties can be useful in various applications like the calibration of a sensor, radar design and as an addition to library database. One can infer the presences of water from the soil permittivity measurement. There are a few techniques in literature for measuring permittivity of the soil. The soil is dielectric in nature and the soil sample may be represented by an electrical equivalent circuit using few discrete components. During the permittivity measurement, metallic electrodes are used to establish a contact with the soil. The electrode resistance and stray capacitance may be explicitly presented in the soil electrical equivalent circuit. In this article, we have attempted to analyze the effect of electrode resistance and that of the stray capacitance on the dielectric permittivity and conductivity of the soil and the simulation results are shown for three different soil equivalent circuits.
\end{abstract}

Keywords: Electrode, Equivalent Circuit, Permittivity, Soil, Stray capacitance

\section{INTRODUCTION AND MOTIVATION}

The study of electromagnetic properties of soil can be useful in RADAR application and also for calibration of a sensor. Such a sensor can be very useful in agriculture and also for in-situ measurement of water ice on the moon and similar planetary applications. Dielectric measurements are widely used for estimation of water content in the environmental substance. Various methods for soil moisture recognition like gravimetric technique, radioactive technique, capacitive technique, conductivity technique and soil suction technique are found in [1]. Many methods are found in the literature for soil property measurement and also for detection of water in the soil [2-9]. Olchawa and Kumor [10] have used the Time Domain Reflectometry (TDR) method to measure dielectric constant of polluted soil to estimate diesel oil content. Bittelli et al. [11] have used the TDR method in conductive soil for moisture measurement. Gong et al. [12] have used TDR method to check the effect of soil bulk density, clay content and temperature on soil moisture measurement. Savi and Maio [13] have carried out sensitivity study for dielectric permittivity estimation using TDR method. Noborio et al. [14] have given soil electrical conductivity and water content in a ridge-furrow tilled loamy sand irrigated with a salt solution by TDR. Chen et al. [15] have given TDR surface reflections for dielectric constant in highly conductive soils. Evett [16] has also given soil water measurement using TDR. Stacheder et al. [17] used four different sensors to measure snow moisture content using dielectric permittivity. Rao and Singh [18] used TDR and capacitance method for soil moisture measurement and gave comparison of both the methods. Skierucha and Wilczek [19] have given FDR sensor for measuring complex soil dielectric permittivity in the 10-500 MHz frequency range. Kyeong-Hwan et al. [20] have given dielectric permittivity sensor for simultaneous measurement of multiple soil properties.

Using the TDR or capacitive sensor one can find the capacitance of the soil and the dielectric permittivity and conductivity may be derived from the capacitance. The soil sample may be represented by an electrical equivalent circuit using discrete components [21]. Pabari et al. [22] have given a simplified electrical circuit for soil and lunar soil simulant JSC-1A testing using WISN. Macdonald [23] has given various electrical circuits based on electrochemical impedance spectroscopy. Katsube et al. [24] have given a structure of mineralized rock for landmine detection. Rock may also be represented by an electrical circuit [25]. Bekhit and Khalil [26] gave electrical circuit for moist limestone. Lampela et al. [27] used electrical circuit for the quartz sand soil and found the impedance for different moisture contents. During the permittivity and conductivity measurement, the metallic electrodes are used to establish a contact with the soil. The electrodes carry signal for the measurement of the soil property. The electrode resistance and stray capacitance may explicitly be represented in the soil electrical equivalent circuit. One may ignore the electrode resistance and stray capacitance during the soil testing. In this article, we have taken three soil circuits and tried to check the effect of electrode resistance and stray capacitance on the soil property during the simplification.

The rest of paper is organized as follows. Section 2 describes basics of impedance, permittivity, and conductivity and also gives various relationships. Section 3 refers three soil electrical equivalent circuits and gives their impedances. Section 4 shows the simulation results using MATHEMATICA [28] and the paper ends with summary and conclusion. 
IOSR Journal of Engineering

Apr. 2012, Vol. 2(4) pp: 837-843

\section{THEORECTICAL BACKGROUND}

Since the soil electrical equivalent circuit may be represented by few discrete components during the soil testing, various components arising in this work are described as follows.

2.1 Electrode Resistance: For soil testing, one needs to use the metallic electrodes to establish the contact with the soil sample. Such electrodes can have a finite amount of resistance with values ranging typically from about $1 \Omega$ to $3 \Omega$. The electrode resistance may create some effect on the permittivity and/or conductivity.

2.2 Stray Capacitance: Any two adjacent conductors can be considered a capacitor, although the capacitance would be small, unless the conductors are very close to each other with longer distance or with larger area. This, often unwanted, effect is termed as stray capacitance. The stray capacitance can cause the signals to leak between otherwise isolated circuits and it can be a limiting factor for proper functioning of circuits at high frequency.

\subsection{Relationship between Impedance, Permittivity and Conductivity}

Impedance is a complex quantity and is given by the equation below [29]

$$
\boldsymbol{Z}=Z_{\text {real }}+j Z_{\text {imag }}
$$

where $j^{2}=-1$.

The relative dielectric permittivity is a complex function and it is defined by the equation [30]

$$
\varepsilon=\varepsilon^{\prime}+j \varepsilon^{\prime \prime}
$$

where $\varepsilon$ is the total relative permittivity

$\varepsilon^{\prime}$ is the real relative permittivity and it is related to the stored energy within the medium

$\varepsilon^{\prime \prime}$ is the imaginary relative permittivity and it is related to the dissipation (or loss) of energy within the medium.

The real relative permittivity shows the relation with imaginary impedance as [22]

$$
\varepsilon^{\prime}=-\frac{Z_{\text {imag }}}{g \omega \varepsilon_{0}\left(Z_{\text {real }}^{2}+Z_{\text {img }}^{2}\right)}
$$

The imaginary relative permittivity is related with real impedance by the equation [22]

$$
\varepsilon^{\prime \prime}=\frac{Z_{\text {real }}}{g \omega \varepsilon_{0}\left(Z_{\text {real }}^{2}+Z_{\text {img }}^{2}\right)}
$$

The real and imaginary conductivity are related to the relative dielectric permittivity by the equations below [22]

and

$$
\sigma^{\prime}=\omega \varepsilon_{0} \varepsilon^{\prime \prime}
$$

\section{SOIL ELECTRICAL EQUIVALENT CIRCUIT}

Soil can be represented by an electrical equivalent circuit using few discrete components. It is known that the soil has dielectric nature and so, it can be represented by a bulk capacitor and resistor in parallel. Pabari et al. [22] used a simplified electrical circuit for the detection of water in soil and lunar soil simulant JSC-1A using the WISN. Lampela et al. [27] used electrical circuit for the quartz sand soil and found the impedances for different moisture contents. Here, we have referred three different soil electrical circuits, which may be used during the measurements. Circuit 1 shown in Figure 1 represents the simplified electrical equivalent soil circuit in which $C$ represents the dielectric nature of the soil and $R$ represents the leakage in the capacitor. 
IOSR Journal of Engineering

Apr. 2012, Vol. 2(4) pp: 837-843

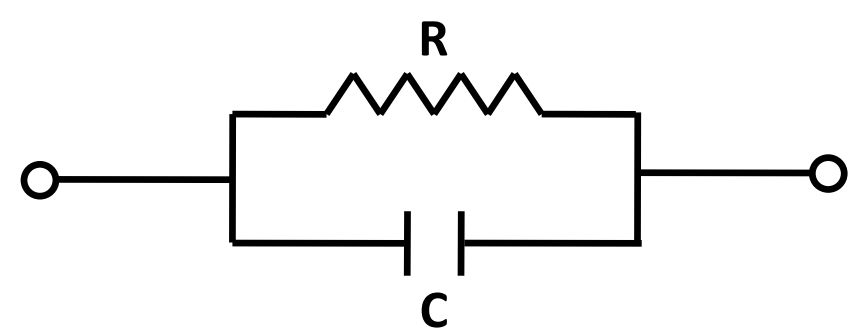

Figure 1: Simplest soil electrical equivalent circuit 1 [21, 22]

Both capacitor and resistor are in parallel in the circuit shown in Figure 1, so the impedance is given by,

$$
\frac{1}{Z}=\frac{1}{R}+\frac{1}{X_{C}}
$$

Simplifying the Equation (7-a), we get

$$
\boldsymbol{Z}_{\mathbf{1}}=\frac{R}{1+R^{2} \omega^{2} C^{2}}-j \frac{R^{2} \omega C}{1+R^{2} \omega^{2} C^{2}}
$$

From which we get the real and imaginary parts of the impedance as [22]

and

$$
\begin{aligned}
& Z_{1 \text { real }}=\frac{R}{1+R^{2} \omega^{2} C^{2}} \\
& Z_{1 \text { imag }}=\frac{-R^{2} \omega C}{1+R^{2} \omega^{2} C^{2}}
\end{aligned}
$$

respectively.

Circuit 2 shown in Figure 2 represents the same structure as that of the circuit 1 in Figure 1, except the addition of the total electrode resistance $\left(R_{e}\right)$ in series.

Because the second circuit has same structure except the series resistance, we add the electrode resistance in the impedance of the first circuit and get

$$
Z_{2}=\frac{R}{1+R^{2} \omega^{2} C^{2}}+R_{e}-j \frac{R^{2} \omega C}{1+R^{2} \omega^{2} C^{2}}
$$

Simplifying Equation (8-a),

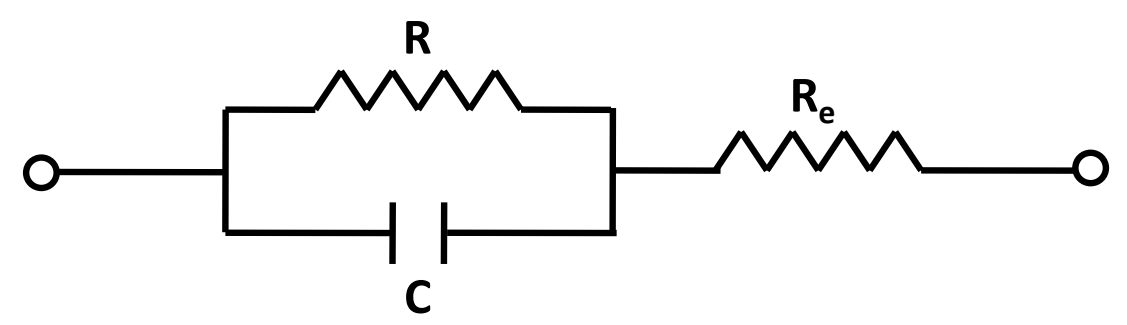

Figure 1: Soil electrical equivalent circuit 2 with electrode resistance included [24-25]

$$
Z_{2}=\frac{R+R_{e}\left(1+R^{2} \omega^{2} C^{2}\right)}{1+R^{2} \omega^{2} C^{2}}-j \frac{R^{2} \omega C}{1+R^{2} \omega^{2} C^{2}}
$$

Circuit 3 depicted in Figure 3 shows the addition of stray capacitor in parallel, as compared to the circuit 2. In Figure 3, $C_{s}$ represents the stray capacitor between two parallel electrodes. 
IOSR Journal of Engineering

Apr. 2012, Vol. 2(4) pp: 837-843

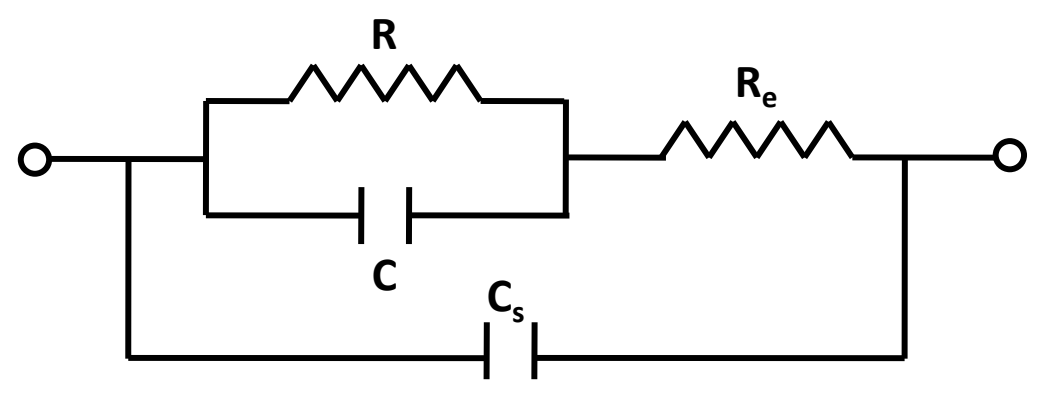

Figure 3: Soil electrical equivalent circuit considering electrode resistance and stray capacitance [27]

Now, the capacitor is in parallel and so, $Z_{3}=Z_{2} \| C_{s}$

Finally, we get real and imaginary parts of the impedance of circuit 3 as

$$
\begin{aligned}
Z_{3 \text { real }}= & \frac{R}{C^{2} C_{S}^{2}\left(\omega^{4} R^{2}+\frac{\omega^{4}}{C^{2}}\right)\left(\left(R_{e}+\frac{R}{\omega^{2} C^{2}\left(R^{2}+\frac{1}{\omega^{2} C^{2}}\right)}\right)^{2}+\left(-\frac{1}{\omega C_{S}}-\frac{R^{2}}{\omega C\left(R^{2}+\frac{1}{\omega^{2} C^{2}}\right)}\right)^{2}\right)}+ \\
& \frac{\omega_{e}^{2} C_{S}{ }^{2}\left(\left(R_{e}+\frac{R}{\omega^{2} C^{2}\left(R^{2}+\frac{1}{\omega^{2} C^{2}}\right)}\right)^{2}+\left(-\frac{1}{\omega C_{S}}-\frac{R^{2}}{\omega C\left(R^{2}+\frac{1}{\omega^{2} C^{2}}\right)}\right)^{2}\right)}{}
\end{aligned}
$$

and

$$
\begin{aligned}
& Z_{\text {aimag }}=-\frac{R^{2}}{\omega^{5} C^{4} C_{s}\left(R^{2}+\frac{1}{\omega^{2} c^{2}}\right)^{2}\left(\left(R_{2}+\frac{R}{\omega^{2} c^{2}\left(R^{2}+\frac{1}{\omega^{2} c^{2}}\right)}\right)^{2}+\left(-\frac{1}{\omega C_{s}}-\frac{R^{2}}{\omega c\left(R^{2}+\frac{1}{\omega^{2} c^{2}}\right)}\right)^{2}\right.}-
\end{aligned}
$$

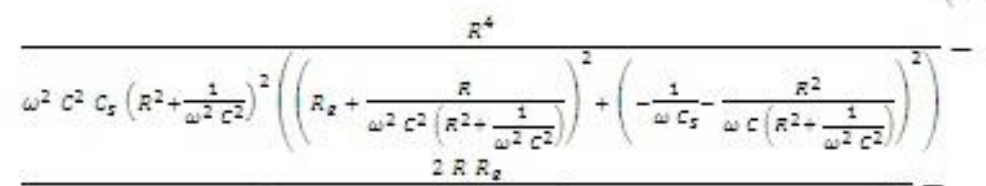

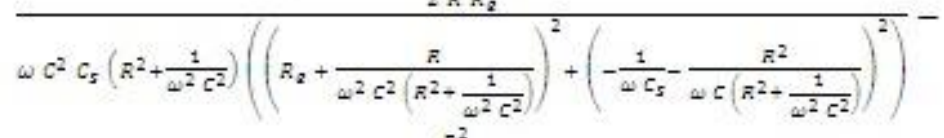

$$
\begin{aligned}
& R^{2} \\
& \overline{\omega C C_{S}\left(R^{2}+\frac{1}{\omega^{2} c^{2}}\right)\left(\left(R_{2}+\frac{R}{\omega^{2} c^{2}\left(R^{2}+\frac{1}{\omega^{2} c^{2}}\right)}\right)^{2}+\left(-\frac{1}{\omega C_{S}}-\frac{R^{2}}{\omega c\left(R^{2}+\frac{1}{\omega^{2} c^{2}}\right)}\right)^{2}\right)}
\end{aligned}
$$

\section{MATHEMATICA SIMULATION RESULTS}

We have carried out simulations of the three soil circuits in the Mathematica [28] software and compared the results based on soil permittivity and conductivity. Considering the practical aspects of the dielectric permittivity and conductivity of the dry soil, we have chosen the values of the bulk as $R=20 M \Omega$ and $C=3 p F$ in the simulations of all the circuits. The soil permittivity and the conductivity based on bulk parameters are shown in Figure 4 (a) and Figure 4 (b), respectively for considering the circuit 1 , circuit 2 or circuit 3. Considering the circuit 2, Figure 5 (a) depicts the three dimensional plot of real relative permittivity versus frequency and the electrode resistance, while Figure 5 (b) depicts the three dimensional plot of conductivity versus frequency and the electrode resistance. Considering the circuit 3, Figure 6 (a) shows the three dimensional plot of real relative permittivity versus frequency and the stray capacitance, while Figure 6 (b) shows the three dimensional plot of conductivity versus frequency and the stray capacitance.

ISSN: $2250-3021$ 


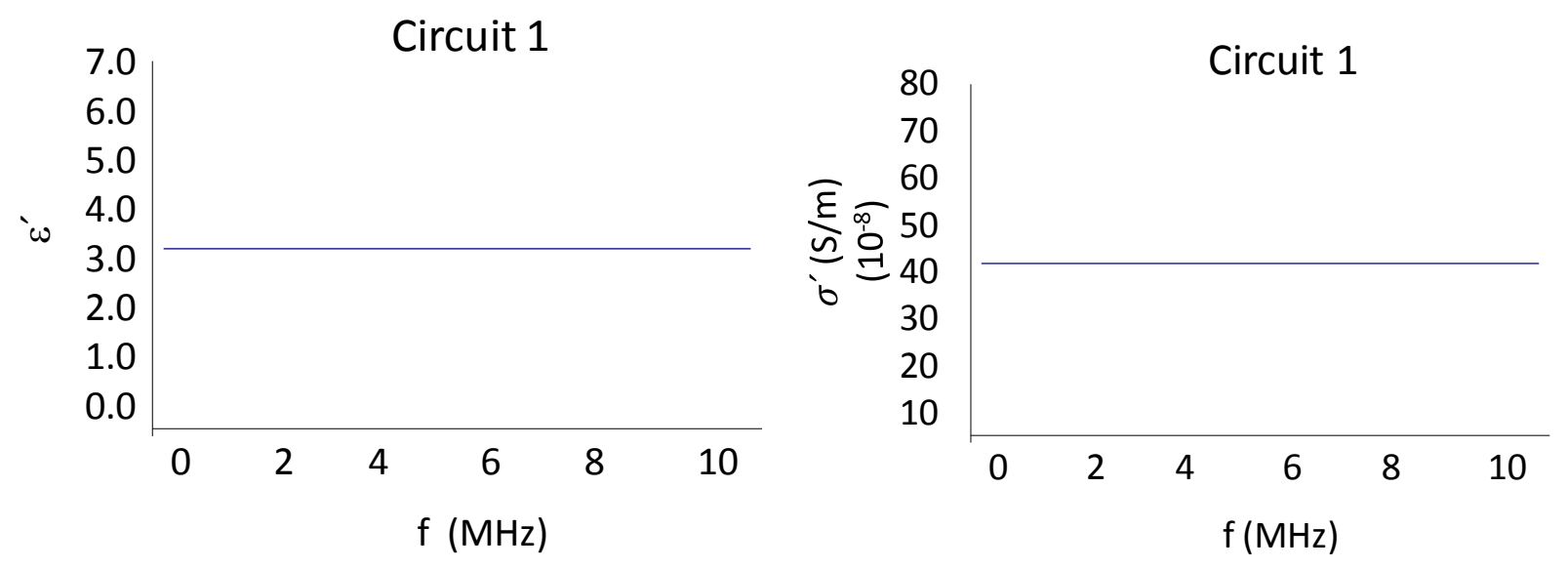

Figure 4: (a) Plot of real relative permittivity vs. frequency and (b) plot of conductivity vs. frequency for circuit 1
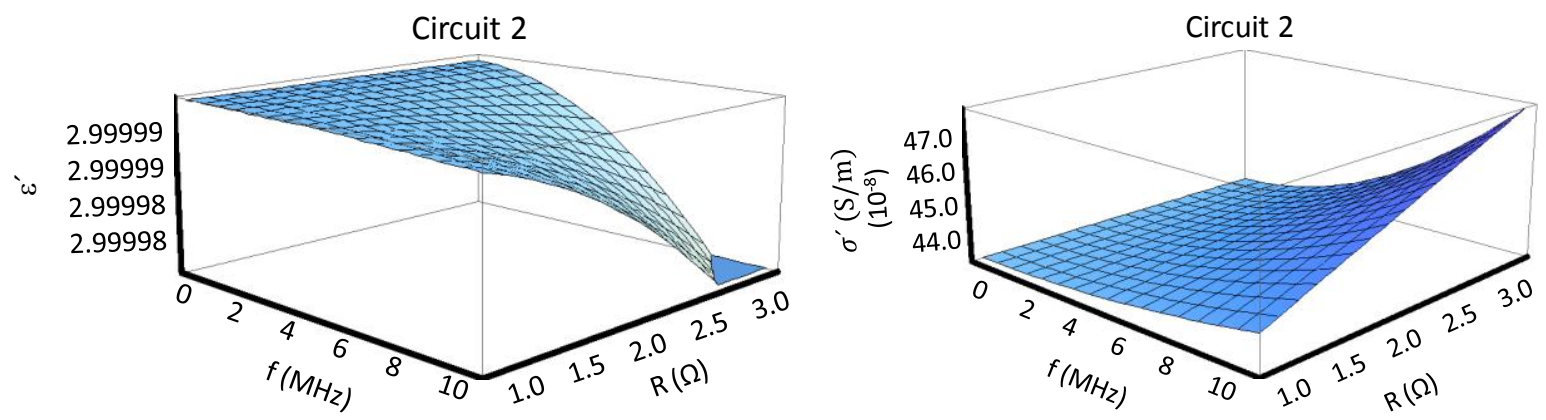

Figure 5: (a) Plot of real relative permittivity vs. electrode resistance and (b) plot of conductivity vs. electrode resistance for circuit 2
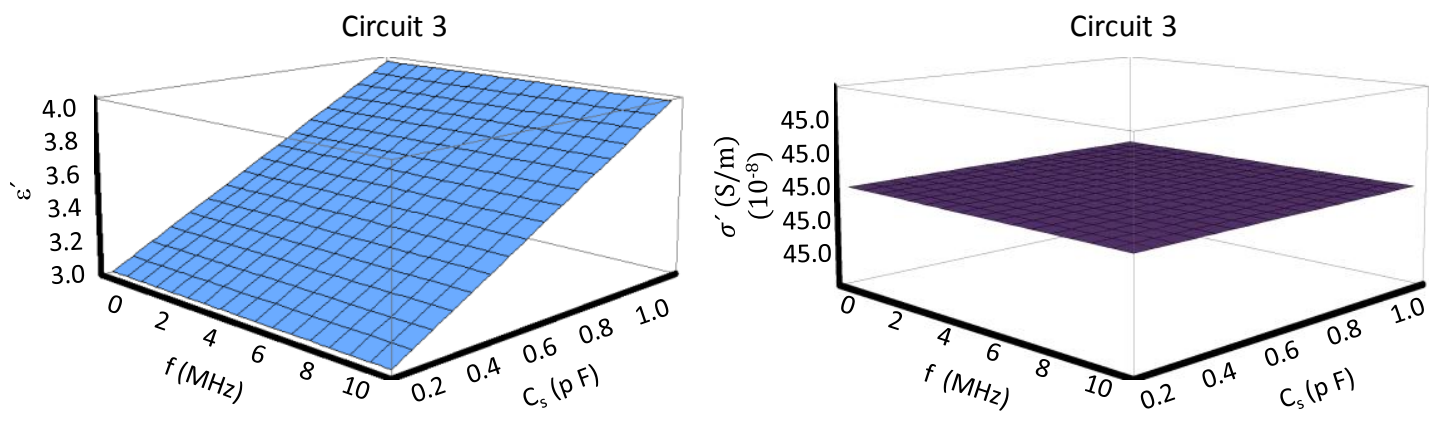

Figure 6: (a) Plot of real relative permittivity vs. stray capacitance and (b) plot of conductivity vs. stray capacitance for circuit 3

\section{DISCUSSION}

5.1 Effect of Electrode Resistance on Permittivity and Conductivity:

By comparing the first (circuit 1) and the second (circuit 2) circuits, one can observe that when the electrode resistance is varied from $1 \Omega$ to $3 \Omega$, there is no appreciable change in case of soil permittivity. Hence, one can ignore the electrode resistance during the permittivity measurement. However, as the value of the electrode resistance is changed, the value of the soil conductivity also changes by a very small amount in the frequency range from $1 \mathrm{MHz}$ to $10 \mathrm{MHz}$.

\subsection{Effect of Stray Capacitance on Permittivity and Conductivity}

By comparing circuit 3 and circuit 1 , one can observe that when the stray capacitance is varied from $0.1 \mathrm{pF}$ to $1 \mathrm{pF}$, there is no appreciable change in case of soil conductivity. Hence, one may ignore the stray capacitance during the conductivity measurement. However, as the value of the stray capacitance is changed from $0.1 \mathrm{pF}$ to $1 \mathrm{pF}$, the value of the soil permittivity 
IOSR Journal of Engineering

Apr. 2012, Vol. 2(4) pp: 837-843

also changes by about $33.3 \%$, typically in the frequency range from $1 \mathrm{MHz}$ to $10 \mathrm{MHz}$. Table 1 shows the effect of electrode resistance on the soil conductivity for circuit 2 and also for circuit 3 (with any value of stray capacitance). Table 2 shows the effect of stray capacitance on the soil permittivity for circuit 3 and also for circuit 2 (without stray capacitance). The error in soil conductivity due to change in the electrode resistance is plotted in Figure 7 (a) and the error in soil permittivity due to change in stray capacitance is plotted in Figure 7 (b). One can observe from Figure 7 (a) and Figure (b) that the error in the soil conductivity is nonlinear with respect to the electrode resistance while the error in soil permittivity varies linearly with respect to the stray capacitance.

Table 1: Variation in conductivity for various values of electrode resistance in circuit 2 and also in circuit 3 with any $\mathrm{C}_{\mathrm{s}}$

\begin{tabular}{|c|c|c|c|}
\hline $\begin{array}{c}\mathbf{f} \\
(\mathbf{M H z})\end{array}$ & $\begin{array}{c}\boldsymbol{\sigma}^{\prime}\left(\text { for } \mathbf{R}_{\mathrm{e}}=\mathbf{1} \mathbf{\Omega}\right) \\
\left(\mathbf{1 0}^{-\mathbf{8}} \boldsymbol{S} / \boldsymbol{m}\right)\end{array}$ & $\begin{array}{c}\boldsymbol{\sigma}^{\prime}\left(\text { for } \mathbf{R}_{\mathrm{e}}=\mathbf{2} \mathbf{\Omega}\right) \\
\left(\mathbf{1 0}^{-\mathbf{8}} \boldsymbol{S} / \boldsymbol{m}\right)\end{array}$ & $\begin{array}{c}\boldsymbol{\sigma}^{\prime}\left(\text { for } \mathbf{R}_{\mathrm{e}}=\mathbf{3} \mathbf{\Omega}\right) \\
\left(\mathbf{1 0}^{-\mathbf{8}} \boldsymbol{S} / \boldsymbol{m}\right)\end{array}$ \\
\hline 1.0 & 44.278 & 44.2859 & 44.2939 \\
\hline 2.5 & 44.3198 & 44.3696 & 44.4194 \\
\hline 5.0 & 44.4692 & 44.6684 & 44.8676 \\
\hline 7.5 & 44.7182 & 45.1665 & 45.6147 \\
\hline 10.0 & 45.0669 & 45.8637 & 46.6606 \\
\hline
\end{tabular}

Table 2: Variation in permittivity for various values of stray capacitance in circuit 3 and also for circuit 2 in the absence of stray capacitance

\begin{tabular}{|c|c|c|c|c|}
\hline \multirow{2}{*}{$\begin{array}{c}\mathbf{f} \\
(\mathbf{M H z})\end{array}$} & For circuit 2 & \multicolumn{3}{|c|}{ For circuit 3 } \\
\cline { 2 - 5 } & $\boldsymbol{\varepsilon}^{\prime}\left(\right.$ for any $\mathbf{R}_{\mathbf{e}}$ ) & $\begin{array}{c}\boldsymbol{\varepsilon}^{\prime}\left(\text { for any } \mathbf{R}_{\mathbf{e}} \text { and }\right. \\
\left.\mathbf{C}_{\mathbf{s}}=\mathbf{0 . 1} \mathbf{~ p F}\right)\end{array}$ & $\begin{array}{c}\boldsymbol{\varepsilon}^{\prime}\left(\text { for } \text { any } \mathbf{R}_{\mathbf{e}} \text { and }\right. \\
\left.\mathbf{C}_{\mathbf{s}}=\mathbf{0 . 5} \mathbf{~ p F}\right)\end{array}$ & $\begin{array}{c}\boldsymbol{\varepsilon}^{\prime}\left(\text { for any } \mathbf{R}_{\mathbf{e}} \text { and }\right. \\
\mathbf{C}_{\mathbf{s}}=\mathbf{1} \mathbf{~ p F} \text { ) }\end{array}$ \\
\hline 1.0 & $\sim 3$ & 3.1 & 3.5 & 4 \\
\hline 2.5 & $\sim 3$ & 3.1 & 3.5 & 4 \\
\hline 5.0 & $\sim 3$ & 3.1 & 3.5 & 4 \\
\hline 7.5 & $\sim 3$ & 3.1 & 3.5 & 4 \\
\hline 10.0 & $\sim 3$ & 3.1 & 3.5 & 4 \\
\hline
\end{tabular}
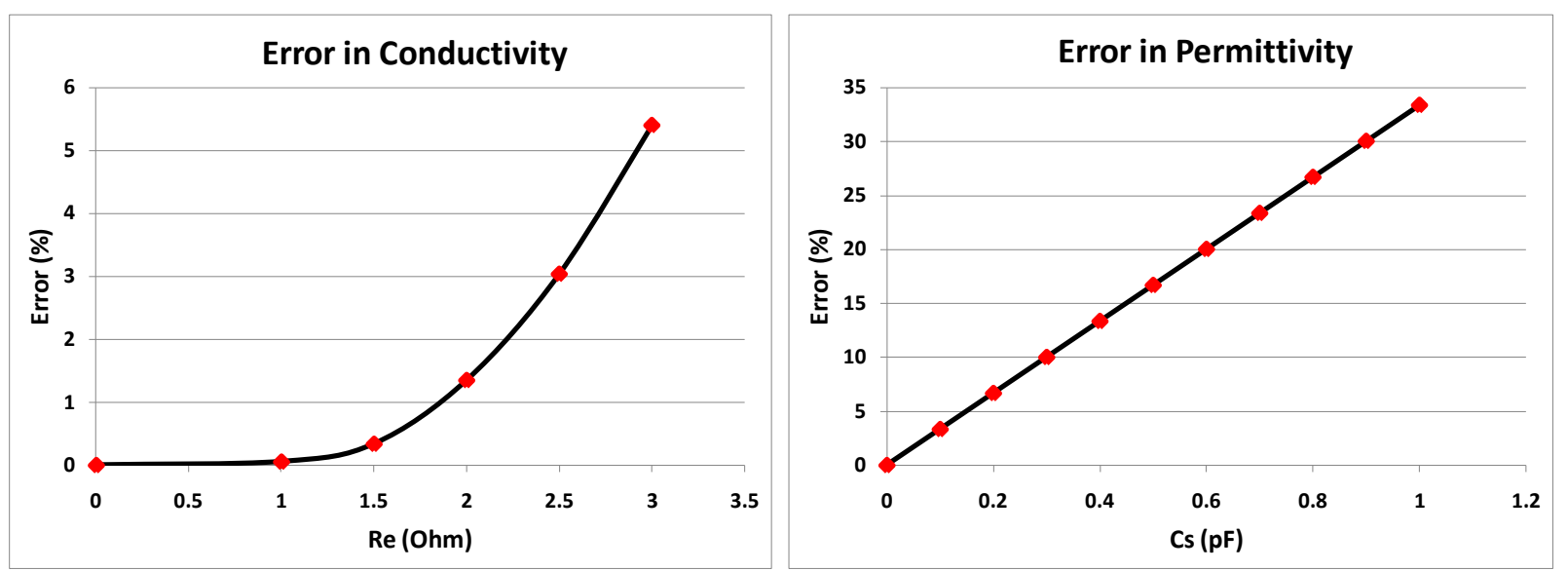

Figure 7: (a) Error in conductivity due to electrode resistance and (b) error in permittivity due to stray capacitance 
IOSR Journal of Engineering

Apr. 2012, Vol. 2(4) pp: 837-843

\section{SUMMARY AND CONCLUSION}

In this paper, we have derived the impedances of the three soil electrical equivalent circuits which may be used in obtaining the soil properties. The three circuits have been compared and the effects of the electrode resistance and the stray capacitance are checked on the measurements of the soil permittivity and the conductivity. The error in soil conductivity is nonlinear with respect to the electrode resistance and there is no effect of electrode resistance on the soil permittivity. For every multiple of 0.1 $\mathrm{pF}$ stray capacitance, the error in the soil permittivity is increased by $3.33 \%$, while there is no effect on the soil conductivity.

\section{REFERENCES}

[1] Skye instrument limited, "Soil Moisture Measurement Notes". Online, $20^{\text {th }}$ March 22, 2012. Available: http://www.skyeinstruments.com.

[2] Online, 20 ${ }^{\text {th }}$ March 22, 2012. Available:

http://asadl.org/jasa/resource/1/jasman/v77/i4/p1601_s1?isAuthorized=no.

[3] Online, $20^{\text {th }}$ March 22, 2012. Available: http://www.lightningman.com.au/Earthing.pdf.

[4] Online, $20^{\text {th }}$ March 22, 2012. Available: http://en.wikipedia.org/wiki/Capacitive sensing.

[5] Online, $20^{\text {th }}$ March 22, 2012. Available: http://cp.literature.agilent.com/litweb/pdf/5989-4149EN.pdf.

[6] Online, $20^{\text {th }}$ March 22, 2012. Available:

http://www.artisan-scientific.com/info/tektronix measuringcontrolledimpedanceboardswithtdrarticle.pdf

[7] Online, $20^{\text {th }}$ March 22, 2012. Available: http://cp.literature.agilent.com/litweb/pdf/5950-3000.pdf.

[8] Online, 20 ${ }^{\text {th }}$ March 22, 2012. Available:

http://www.experimental-hydrology.net/wiki/index.php?title=Soil_moisture_-_impedance_method_(Theta_Probe)

[9] Online, $5^{\text {th }}$ January, 2012. Available:

http://seismo.berkeley.edu/ rallen/teaching/F04_GEO594_IntroAppGeophys/Lectures/L8.pdf.

[10] Andrzej Olchawa and Maciej Kumor, "Time Domain Reflectometry (TDR) - Measuring Dielectric Constant of Polluted Soil to Estimate Diesel Oil Content", Archives of Hydro-Engineering and Environmental Mechanics, Vol. 55, No. 1-2, 2008, pp. 55-62.

[11] Marco Bittelli, Fiorenzo Salvatorelli and Paola Rossi Pisa, "Correction of TDR-based soil water content measurements in conductive soils", Geoderma, Elsevier, Vol. 143, 2008, pp. 133-142.

[12] Yuanshi Gong, Qiaohong Cao and Zongjia Sun, "The effects of soil bulk density, clay content and temperature on soil water content measurement using time-domain reflectometry”, Hydrological Processes, Wiley InterScience, Vol. 17, 2003, pp. 3601-3614.

[13] P. Savi and I.A. Maio, "Sensitivity Study for Dielectric Permittivity Estimation via TDR Measurements", Online, $16^{\text {th }}$ April 2012. Available: http://www.emc.polito.it/publications/file/cnf-2006-medmicrowave-sensitivity.pdf.

[14] K. Noborio, K. J. McInnes and J. L. Heilman, "Field measurements of soil electrical conductivity and water content by timedomain reflectometry", Computers and Electronics in Agriculture, Elsvier, Vol. 11, Issues 2-3, 1994, pp. 131-142.

[15] Renpeng Chen, Vincent P. Drnevich, Xiong Yu, Robert L. Nowack and Yunmin Chen, "Time Domain Reflectometry Surface Reflections for Dielectric Constant in Highly Conductive Soils”, J. Geotech. Geoenviron. Eng., Vol. 133, 2007, p. 1597.

[16] Steven R. Evett, "Soil Water Measurement by Time Domain Reflectometry", Encyclopedia of Water Science, Marcel Dekker Inc. New York, 2003, pp. 894-898.

[17] Markus Stacheder, Franz Koeniger and Rainer Schuhmann, "New Dielectric Sensors and Sensing Techniques for Soil and Snow Moisture Measurements", Sensors, 2009, Vol. 9, pp. 2951-2967.

[18] B. Hanumantha Rao and D. N. Singh, "Moisture Content Determination by TDR and Capacitance Techniques: A Comparative Study", International Journal of Earth Sciences and Engineering, Vol. 4, No. 6 SPL, 2011, pp. 132-137.

[19] Wojciech Skierucha and Andrzej Wilczek, "A FDR Sensor for Measuring Complex Soil Dielectric Permittivity in the 10500 MHz Frequency Range”, Sensors, Vol. 10, 2010, pp. 3314-3329.

[20] Kyeong-Hwan, Lee Naiqian Zhang, Gerard Kluitenberg, William B. Kuhn and Sanjoy Das, "Dielectric Permittivity Sensor for Simultaneous Measurement of Multiple Soil Properties", ASAE Annual Meeting, American Society of Agricultural and Biological Engineers, Paper No. 041045, 2004.

[21] Online, $20^{\text {th }}$ March, 2012. Available: http://seismo.berkeley.edu/ rallen/teaching/F04_GEO594_IntroAppGeophys/Lectures/L8.pdf.

[22] J. P. Pabari, Y. B. Acharya, U. B. Desai and S. N. Merchant, "Development of Impedance Based, Miniaturized Wireless Water Ice Sensor for Future Planetary Applications", IEEE Transactions on Instrumentation and Measurement, Vol. 61, Issue 2, 2012, pp. 521-529.

[23] J. Ross Macdonald, "Impedance Spectroscopy", Annals of Biomedical Engineering, Vol. 20, 1992, pp. 289-305.

[24] T. J. Katsube, E. Grunsky, Y. Das, R. DiLabio, H. McVairn, S. Connell-Madora, E.Gauthier and N. Scromeda, "Physical model of soil and its implications for landmine detection interferences", Proc. of SPIE, Vol. 6217, 62170N, 2006. 
IOSR Journal of Engineering

Apr. 2012, Vol. 2(4) pp: 837-843

[25] Online, 27 ${ }^{\text {th }}$ March, 2012. Available: http://appliedgeophysics.lbl.gov/dc/em43.pdf.

[26] Marzouk M. Bekhit and Saad A. Khalil, "Electrical Properties of Moist Limestone Samples In The Frequency Range 1Hz$10 \mathrm{~Hz}$ From Abu Rawash Area", Australian Journal of Basic and Applied Sciences, Vol. 1, No. 4, 2007, pp. 741-750.

[27] Mika Lampela, Tapani Repo, Raimo Silvennoinen and Leena Finer, "Equivalent electrical circuit model for system containing quartz sand and purified water", Online, 16 ${ }^{\text {th }}$ April, 2012. Available: http://www.metla.fi/jo/juuristolaboratorio/pdf/Poster6_Lampela\%20et\%20al_Poster\%20in\%20E38\%20Rovaniemi.pdf.

[28] Online, $5^{\text {th }}$ January, 2012. Available: www.wolfram.com/mathematica/.

[29] Online, $5^{\text {th }}$ January, 2012. Available $:$ http://en.wikipedia.org/wiki/Electrical_impedance.

[30] Online, $5^{\text {th }}$ January, 2012. Available : http://en.wikipedia.org/wiki/Dielectric_constant. 